\title{
A NEW SPECTRAL CANCELLATION IN QUANTUM GRAVITY
}

\section{GIAMPIERO ESPOSITO}

INFN, Sezione di Napoli, Complesso Universitario di Monte S. Angelo, Via Cintia, Edificio N', 80126 Napoli, Italy

Università degli Studi di Napoli Federico II, Dipartimento di Scienze Fisiche, Complesso Universitario di Monte S. Angelo, Via Cintia, Edificio N', 80126 Napoli, Italy

E-mail: giampiero.esposito@na.infn.it

\section{GUGLIELMO FUCCI}

Department of Physics, New Mexico Institute of Mining and Technology, Leroy Place 801, Socorro, NM 87801, USA

E-mail: gfucci@nmt.edu

\section{ALEXANDER KAMENSHCHIK}

Dipartimento di Fisica and INFN, Via Irnerio 46, 40126 Bologna, Italy L.D. Landau Institute for Theoretical Physics, Kosygin str. 2, 119334 Moscow, Russia

E-mail: alexander.kamenshchik@bo.infn.it

\section{KLAUS KIRSTEN}

Department of Mathematics, Baylor University, Waco TX 76798, USA

E-mail: Klaus_Kirsten@baylor.edu 
A general method exists for studying Abelian and non-Abelian gauge theories, as well as Euclidean quantum gravity, at one-loop level on manifolds with boundary. In the latter case, boundary conditions on metric perturbations $h$ can be chosen to be completely invariant under infinitesimal diffeomorphisms, to preserve the invariance group of the theory and BRST symmetry. In the de Donder gauge, however, the resulting boundary-value problem for the Laplace type operator acting on $h$ is known to be self-adjoint but not strongly elliptic. The present paper shows that, on the Euclidean four-ball, only the scalar part of perturbative modes for quantum gravity is affected by the lack of strong ellipticity. Interestingly, three sectors of the scalar-perturbation problem remain elliptic, while lack of strong ellipticity is "confined" to the remaining fourth sector. The integral representation of the resulting $\zeta$-function asymptotics on the Euclidean four-ball is also obtained; this remains regular at the origin by virtue of a peculiar spectral identity obtained by the authors. There is therefore encouraging evidence in favour of the $\zeta(0)$ value with fully diff-invariant boundary conditions remaining well defined, at least on the four-ball, although severe technical obstructions remain in general.

2000 MSC. Primary 58J35, 83C45; Secondary 81S40, 81T20.

\section{Introduction}

This paper is motivated by the authors' struggle over many years with an important problem in quantum field theory and spectral geometry, i.e. the functional determinant in Euclidean quantum gravity on manifolds with non-empty boundary. The related open issues are not yet settled, but there is a sufficient amount of new calculations to justify further efforts, as we are going to see shortly.

The subject of boundary effects in quantum field theory (Deutsch and Candelas [1]) has always received a careful consideration in the literature by virtue of very important physical and mathematical motivations, that can be summarized as follows.

(i) Boundary data play a crucial role in the functional-integral approach (DeWitt [2]), in the quantum theory of the early universe (Hartle and Hawking, Hawking [3]) in supergravity (Hawking [4]) and even in string theory (Abouelsaood et al. [5]).

(ii) The way in which quantum fields react to the presence of boundaries is responsible for remarkable physical effects, e.g. the attractive Casimir force among perfectly conducting parallel plates (Bordag et al., Milton, Nesterenko et al. [6]), which can be viewed as arising from differences of zero-point energies of the quantized electromagnetic field.

(iii) The spectral geometry of a Riemannian manifold (Gilkey [7]) with boundary is a fascinating problem where many new results have been derived over the last few years (Kirsten [8], Vassilevich [9]). 
(iv) Boundary terms (Moss [10]) in heat-kernel expansions have become a major subject of investigation in quantum gravity (Avramidi [11]), since they shed new light on one-loop conformal anomalies (Esposito et al., Moss and Poletti [12], Tsoupros [13]) and one-loop divergences (Esposito [14], Esposito et al. [15]).

In our paper we are interested in boundary conditions for metric perturbations that are completely invariant under infinitesimal diffeomorphisms, since they are part of the general scheme according to which the boundary conditions are preserved under the action of the symmetry group of the theory (Barvinsky [16], Moss and Silva [17], Avramidi and Esposito [18]). In field-theoretical language, this means setting to zero at the boundary that part $\pi A$ of the gauge field $A$ that lives on the boundary $\mathcal{B}$ ( $\pi$ being a projection operator):

$$
[\pi A]_{\mathcal{B}}=0
$$

as well as the gauge-fixing functional,

$$
[\Phi(A)]_{\mathcal{B}}=0
$$

and the whole ghost field

$$
[\varphi]_{\mathcal{B}}=0 .
$$

For Euclidean quantum gravity, Eq. (1) reads as

$$
\left[h_{i j}\right]_{\mathcal{B}}=0,
$$

where $h_{i j}$ are perturbations of the induced three-metric. To arrive at the gravitational counterpart of Eqs. (2) and (3), note first that, under infinitesimal diffeomorphisms, metric perturbations $h_{\mu \nu}$ transform according to

$$
\widehat{h}_{\mu \nu} \equiv h_{\mu \nu}+\nabla_{(\mu} \varphi_{\nu)},
$$

where $\nabla$ is the Levi-Civita connection on the background four-geometry with metric $g$, and $\varphi_{\nu} d x^{\nu}$ is the ghost one-form (strictly, our presentation is simplified: there are two independent ghost fields obeying Fermi statistics, and we will eventually multiply by -2 the effect of $\varphi_{\nu}$ to take this into account). In geometric language, the infinitesimal variation $\delta h_{\mu \nu} \equiv \widehat{h}_{\mu \nu}-$ $h_{\mu \nu}$ is given by the Lie derivative along $\varphi$ of the four-metric $g$. For manifolds with boundary, Eq. (5) implies that (Esposito et al. [19], Avramidi et al. [20])

$$
\widehat{h}_{i j}=h_{i j}+\varphi_{(i \mid j)}+K_{i j} \varphi_{0},
$$


where the stroke denotes three-dimensional covariant differentiation tangentially with respect to the intrinsic Levi-Civita connection of the boundary, while $K_{i j}$ is the extrinsic-curvature tensor of the boundary. Of course, $\varphi_{0}$ and $\varphi_{i}$ are the normal and tangential components of the ghost, respectively. By virtue of Eq. (6), the boundary conditions (4) are "gauge invariant", i.e.

$$
\left[\widehat{h}_{i j}\right]_{\mathcal{B}}=0
$$

if and only if the whole ghost field obeys homogeneous Dirichlet conditions, so that

$$
\begin{aligned}
{\left[\varphi_{0}\right]_{\mathcal{B}} } & =0, \\
{\left[\varphi_{i}\right]_{\mathcal{B}} } & =0 .
\end{aligned}
$$

The conditions (8) and (9) are necessary and sufficient since $\varphi_{0}$ and $\varphi_{i}$ are independent, and three-dimensional covariant differentiation commutes with the operation of restriction to the boundary. We are indeed assuming that the boundary $\mathcal{B}$ is smooth and not totally geodesic, i.e. $K_{i j} \neq 0$. However, for totally geodesic boundaries, having $K_{i j}=0$, the condition (8) is no longer necessary.

On imposing boundary conditions on the remaining set of metric perturbations, the key point is to make sure that the invariance of such boundary conditions under the infinitesimal transformations (5) is again guaranteed by (8) and (9), since otherwise one would obtain incompatible sets of boundary conditions on the ghost field. Indeed, on using the DeWittFaddeev-Popov formalism for the 〈out|in〉 amplitudes of quantum gravity, it is necessary to use a gauge-averaging term in the Euclidean action, of the form ${ }^{2}$

$$
I_{g . a .}=\frac{1}{16 \pi G} \int_{\mathcal{M}} \frac{\Phi_{\nu} \Phi^{\nu}}{2 \alpha} \sqrt{\operatorname{det} g} d^{4} x
$$

where $\Phi_{\nu}$ is any functional which leads to self-adjoint (elliptic) operators on metric and ghost perturbations. One then finds that

$$
\delta \Phi_{\mu}(h) \equiv \Phi_{\mu}(h)-\Phi_{\mu}(\widehat{h})=\mathcal{F}_{\mu}^{\nu} \varphi_{\nu},
$$

where $\mathcal{F}_{\mu}{ }^{\nu}$ is an elliptic operator that acts linearly on the ghost field. Thus, if one imposes the boundary conditions

$$
\left[\Phi_{\mu}(h)\right]_{\mathcal{B}}=0
$$


and if one assumes that the ghost field can be expanded in a complete orthonormal set of eigenfunctions $u_{\nu}^{(\lambda)}$ of $\mathcal{F}_{\mu}^{\nu}$ which vanish at the boundary, i.e.

$$
\begin{gathered}
\mathcal{F}_{\mu}^{\nu} u_{\nu}^{(\lambda)}=\lambda u_{\mu}^{(\lambda)}, \\
\varphi_{\nu}=\sum_{\lambda} C_{\lambda} u_{\nu}^{(\lambda)}, \\
{\left[u_{\mu}^{(\lambda)}\right]_{\mathcal{B}}=0,}
\end{gathered}
$$

the boundary conditions (12) are automatically gauge-invariant under the Dirichlet conditions (8) and (9) on the ghost.

Having obtained the general recipe expressed by Eqs. (4) and (12), we can recall what they imply on the Euclidean four-ball. This background is relevant for one-loop quantum cosmology in the limit of small threegeometry on the one hand (Schleich [21]), and for spectral geometry and spectral asymptotics on the other hand $[8,9]$. As shown in Ref. 19, if one chooses the de Donder gauge-fixing functional

$$
\Phi_{\mu}(h)=\nabla^{\nu}\left(h_{\mu \nu}-\frac{1}{2} g_{\mu \nu} g^{\rho \sigma} h_{\rho \sigma}\right),
$$

which has the virtue of leading to an operator of Laplace type on $h_{\mu \nu}$ in the one-loop functional integral, Eq. (12) yields the mixed boundary conditions

$$
\begin{gathered}
{\left[\frac{\partial h_{00}}{\partial \tau}+\frac{6}{\tau} h_{00}-\frac{\partial}{\partial \tau}\left(g^{i j} h_{i j}\right)+\frac{2}{\tau^{2}} h_{0 i}^{\mid i}\right]_{\mathcal{B}}=0,} \\
{\left[\frac{\partial h_{0 i}}{\partial \tau}+\frac{3}{\tau} h_{0 i}-\frac{1}{2} \frac{\partial h_{00}}{\partial x^{i}}\right]_{\mathcal{B}}=0 .}
\end{gathered}
$$

In Refs. 15, 19, the boundary conditions (4), (17) and (18) were used to evaluate the full one-loop divergence of quantized general relativity on the Euclidean four-ball, including all $h_{\mu \nu}$ and all ghost modes. However, the meaning of such a calculation became unclear after the discovery in Ref. 18 that the boundary-value problem for the Laplacian $P$ acting on metric perturbations is not strongly elliptic by virtue of tangential derivatives in the boundary conditions (17) and (18). Moreover, the work by Dowker and Kirsten [22] had proved even earlier, in a simpler case, that the boundaryvalue problem with tangential derivatives is, in general, not strongly elliptic. Strong ellipticity $[8,18]$ is a technical requirement ensuring that a unique smooth solution of the boundary-value problem exists which vanishes at 
infinite geodesic distance from the boundary. If it is fulfilled, this ensures that the $L^{2}$ trace of the heat semigroup $e^{-t P}$ exists, with the associated global heat-kernel asymptotics that yields one-loop divergence and one-loop effective action. However, when strong ellipticity does not hold, the $L^{2}$ trace of $e^{-t P}$ acquires a singular part [18] and hence $\zeta$-function calculations may become ill-defined.

All of this has motivated our analysis, which therefore derives in Sec. 2 the eigenvalue conditions for scalar modes. Section 3 obtains the first pair of resulting scalar-mode $\zeta$-functions and Sec. 4 studies the remaining elliptic and non-elliptic parts of spectral asymptotics. Results and open problems are described in Sec. 5.

\section{Eigenvalue conditions for scalar modes on the four-ball}

On the Euclidean four-ball, which can be viewed as the portion of flat Euclidean four-space bounded by a three-sphere of radius $q$, metric perturbations $h_{\mu \nu}$ can be expanded in terms of hyperspherical harmonics as (Lifshitz and Khalatnikov [23], Esposito et al. [24])

$$
\begin{gathered}
h_{00}(x, \tau)=\sum_{n=1}^{\infty} a_{n}(\tau) Q^{(n)}(x) \\
h_{0 i}(x, \tau)=\sum_{n=2}^{\infty}\left[b_{n}(\tau) \frac{Q_{\mid i}^{(n)}(x)}{\left(n^{2}-1\right)}+c_{n}(\tau) S_{i}^{(n)}(x)\right] \\
h_{i j}(x, \tau)=\sum_{n=3}^{\infty} d_{n}(\tau)\left[\frac{Q_{\mid i j}^{(n)}(x)}{\left(n^{2}-1\right)}+\frac{c_{i j}}{3} Q^{(n)}(x)\right]+\sum_{n=1}^{\infty} \frac{e_{n}(\tau)}{3} c_{i j} Q^{(n)}(x) \\
+\sum_{n=3}^{\infty}\left[f_{n}(\tau)\left(S_{i \mid j}^{(n)}(x)+S_{j \mid i}^{(n)}(x)\right)+k_{n}(\tau) G_{i j}^{(n)}(x)\right]
\end{gathered}
$$

where $\tau \in[0, q]$ and $Q^{(n)}(x), S_{i}^{(n)}(x)$ and $G_{i j}^{(n)}(x)$ are scalar, transverse vector and transverse-traceless tensor hyperspherical harmonics, respectively, on a unit three-sphere with metric $c_{i j}$. By insertion of the expansions (19)-(21) into the eigenvalue equation for the Laplacian acting on $h_{\mu \nu}$, and by setting $\sqrt{E} \rightarrow i M$, which corresponds to a rotation of contour in the $\zeta$-function analysis (Barvinsky et al. [25]) one finds the modes as linear combinations of modified Bessel functions of first kind. Modified Bessel functions of the second kind are not included to ensure regularity at the 
origin $\tau=0$. For details, we refer the reader to the work by Esposito et al. [26].

The boundary conditions (4), (17), (18), (8), (9), jointly with the modeexpansions on the four-ball, can be used to obtain homogeneous linear systems that yield, implicitly, the eigenvalues of our problem. The conditions for finding non-trivial solutions of such linear systems are given by the vanishing of the associated determinants; these yield the eigenvalue conditions $\delta(E)=0$, i.e. the equations obeyed by the eigenvalues by virtue of the boundary conditions. For the purpose of a rigorous analysis, we need the full expression of such eigenvalue conditions for each set of coupled modes. Upon setting $\sqrt{E} \rightarrow i M$, we denote by $D(M q)$ the counterpart of $\delta(E)$, bearing in mind that, strictly, only $\delta(E)$ yields implicitly the eigenvalues, while $D(M q)$ is more convenient for $\zeta$-function calculations [25].

In particular, we here focus on scalar modes (for the whole set of modes, see again the work in Ref. 26). For all $n \geq 3$, coupled scalar modes $a_{n}, b_{n}, d_{n}, e_{n}$ are ruled by a determinant reading as

$$
D_{n}(M q)=\operatorname{det} \rho_{i j}(M q),
$$

with degeneracy $n^{2}$, where $\rho_{i j}$ is a $4 \times 4$ matrix with entries (hereafter, $I_{n}$ are modified Bessel functions of first kind)

$$
\begin{gathered}
\rho_{11}=I_{n}(M q)-M q I_{n}^{\prime}(M q), \rho_{12}=M q I_{n}^{\prime}(M q), \\
\rho_{13}=(2-n) I_{n-2}(M q)+M q I_{n-2}^{\prime}(M q), \\
\rho_{14}=(2+n) I_{n+2}(M q)+M q I_{n+2}^{\prime}(M q), \\
\rho_{21}=-\left(n^{2}-1\right) I_{n}(M q), \rho_{22}=2 M q I_{n}^{\prime}(M q)+6 I_{n}(M q), \\
\rho_{23}=2(n+1) M q I_{n-2}^{\prime}(M q)-\left(n^{2}-6 n-7\right) I_{n-2}(M q), \\
\rho_{24}=-2(n-1) M q I_{n+2}^{\prime}(M q)-\left(n^{2}+6 n-7\right) I_{n+2}(M q), \\
\rho_{31}=0, \rho_{32}=-I_{n}(M q), \\
\rho_{33}=\frac{(n+1)}{(n-2)} I_{n-2}(M q), \rho_{34}=\frac{(n-1)}{(n+2)} I_{n+2}(M q), \\
\rho_{41}=3 I_{n}(M q), \rho_{42}=-2 I_{n}(M q), \rho_{43}=-I_{n-2}(M q), \rho_{44}=-I_{n+2}(M q) .
\end{gathered}
$$

The hardest part of our analysis is the investigation of the equation obtained by setting to zero the determinant (22). For this purpose, we first exploit 
the recurrence relations among $I_{n}, I_{n+1}$ and $I_{n}^{\prime}$ to find (from now on, $w \equiv$ $M q)$

$$
\begin{aligned}
& \rho_{11}=I_{n}(w)-w I_{n}^{\prime}(w), \rho_{12}=w I_{n}^{\prime}(w), \rho_{13}=w I_{n}^{\prime}(w)+n I_{n}(w), \\
& \rho_{14}=w I_{n}^{\prime}(w)-n I_{n}(w) \text {, } \\
& \rho_{21}=-\left(n^{2}-1\right) I_{n}(w), \rho_{22}=2\left(w I_{n}^{\prime}(w)+3 I_{n}(w)\right), \\
& \rho_{23}=(n+1)\left\{\left[3(n+1)+\frac{2 n(n-1)(n+3)}{w^{2}}\right] I_{n}(w)\right. \\
& \left.+2\left[w+\frac{(n-1)(n+3)}{w}\right] I_{n}^{\prime}(w)\right\} \\
& \rho_{24}=(n-1)\left\{\left[3(n-1)+\frac{2 n(n+1)(n-3)}{w^{2}}\right] I_{n}(w)\right. \\
& \left.-2\left[w+\frac{(n+1)(n-3)}{w}\right] I_{n}^{\prime}(w)\right\} \text {, } \\
& \rho_{31}=0, \rho_{32}=-I_{n}(w), \\
& \rho_{33}=\frac{(n+1)}{(n-2)}\left[\left(1+\frac{2 n(n-1)}{w^{2}}\right) I_{n}(w)+\frac{2(n-1)}{w} I_{n}^{\prime}(w)\right], \\
& \rho_{34}=\frac{(n-1)}{(n+2)}\left[\left(1+\frac{2 n(n+1)}{w^{2}}\right) I_{n}(w)-\frac{2(n+1)}{w} I_{n}^{\prime}(w)\right], \\
& \rho_{41}=3 I_{n}(w), \rho_{42}=-2 I_{n}(w), \\
& \rho_{43}=-\left(1+\frac{2 n(n-1)}{w^{2}}\right) I_{n}(w)-\frac{2(n-1)}{w} I_{n}^{\prime}(w), \\
& \rho_{44}=-\left(1+\frac{2 n(n+1)}{w^{2}}\right) I_{n}(w)+\frac{2(n+1)}{w} I_{n}^{\prime}(w) .
\end{aligned}
$$

The resulting determinant, despite its cumbersome expression, can be studied by introducing the variable

$$
y \equiv \frac{I_{n}^{\prime}(w)}{I_{n}(w)}
$$

which leads to

$$
D_{n}(w)=\frac{48 n\left(1-n^{2}\right)}{\left(n^{2}-4\right)} I_{n}^{4}(w)\left(y-y_{1}\right)\left(y-y_{2}\right)\left(y-y_{3}\right)\left(y-y_{4}\right),
$$


where

$$
y_{1} \equiv-\frac{n}{w}, y_{2} \equiv \frac{n}{w}, y_{3} \equiv-\frac{n}{w}-\frac{w}{2}, y_{4} \equiv \frac{n}{w}-\frac{w}{2},
$$

and hence

$$
\begin{aligned}
& \quad \frac{\left(n^{2}-4\right)}{48 n\left(1-n^{2}\right)} D_{n}(w)=\left(I_{n}^{\prime}(w)+\frac{n}{w} I_{n}(w)\right)\left(I_{n}^{\prime}(w)-\frac{n}{w} I_{n}(w)\right) \\
& \cdot\left(I_{n}^{\prime}(w)+\left(\frac{w}{2}+\frac{n}{w}\right) I_{n}(w)\right)\left(I_{n}^{\prime}(w)+\left(\frac{w}{2}-\frac{n}{w}\right) I_{n}(w)\right) .
\end{aligned}
$$

\section{First pair of scalar-mode $\zeta$-functions}

In our problem, the differential operator under investigation is the Laplacian on the Euclidean four-ball acting on metric perturbations. The boundary conditions for vector, tensor and ghost modes correspond to a familiar mixture of Dirichlet and Robin boundary conditions for which an integral representation of the $\zeta$-function and heat-kernel coefficients are immediately obtained. New features arise instead from Eq. (44), that gives rise to four different $\zeta$-functions. On studying the first line of Eq. (44), we exploit the Cauchy integral formula to express the power $-s$ of the eigenvalues and hence turn the $\zeta$-function

$$
\zeta_{A}^{ \pm}(s) \equiv \sum_{n=3}^{\infty} n^{2} \lambda_{A^{ \pm}}^{-s}
$$

into an integral, i.e. we use

$$
\sum_{l=1}^{\infty} x_{l}^{-s}=\int_{\gamma} d x x^{-s} \frac{d}{d x} \log H_{n}(x),
$$

where $\gamma$ encloses the zeros $x_{1}, x_{2}, \ldots, x_{\infty}$ of the function $H_{n}$, which here equals $J_{n}^{\prime}(x) \pm \frac{n}{x} J_{n}(x)$. Such a combination of $J_{n}$ and $J_{n}^{\prime}$ is proportional to the power of degree $\left(\beta_{ \pm}-1\right)$ of the independent variable multiplied by an infinite product, with $\beta_{+}(n) \equiv n, \beta_{-}(n) \equiv n+2$. Only the infinite product encodes information on the countable infinity of non-vanishing zeros, and hence one should divide $x J_{n}^{\prime}(x) \pm n J_{n}(x)$ by $x^{\beta_{ \pm}}$. Last, rotation of contour to the imaginary axis (Dowker and Kirsten [22], Bordag et al. [27]), which brings in modified Bessel functions $I_{n}$, jointly with setting $w=z n$, leads to the following integral formula:

$$
\zeta_{A}^{ \pm}(s) \equiv \frac{(\sin \pi s)}{\pi} \sum_{n=3}^{\infty} n^{-(2 s-2)} \int_{0}^{\infty} d z z^{-2 s} \frac{\partial}{\partial z} \log \left[\frac{\left(z n I_{n}^{\prime}(z n) \pm n I_{n}(z n)\right)}{z^{\beta_{ \pm}(n)}}\right] .
$$


The uniform asymptotic expansion of modified Bessel functions and their first derivatives (see Appendix) can be used to find (hereafter $\tau=$ $\left.\tau(z) \equiv\left(1+z^{2}\right)^{-\frac{1}{2}}\right)$

$$
z n I_{n}^{\prime}(z n) \pm n I_{n}(z n) \sim \frac{n}{\sqrt{2 \pi n}} \frac{e^{n \eta}}{\sqrt{\tau}}(1 \pm \tau)\left(1+\sum_{k=1}^{\infty} \frac{p_{k, \pm}(\tau)}{n^{k}}\right),
$$

where (see Eqs. (139) and (141) in the Appendix for the functions $u_{k}$ and $\left.v_{k}\right)$

$$
p_{k, \pm}(\tau) \equiv(1 \pm \tau)^{-1}\left(v_{k}(\tau) \pm \tau u_{k}(\tau)\right),
$$

for all $k \geq 1$, and

$$
\log \left(1+\sum_{k=1}^{\infty} \frac{p_{k, \pm}(\tau)}{n^{k}}\right) \sim \sum_{k=1}^{\infty} \frac{T_{k, \pm}(\tau)}{n^{k}}
$$

Thus, the $\zeta$-functions (45) obtain, from the first pair of round brackets in Eq. (46), the contributions (cf. Ref. 22)

$$
\begin{aligned}
& A_{+}(s) \equiv \sum_{n=3}^{\infty} n^{-(2 s-2)} \frac{(\sin \pi s)}{\pi} \int_{0}^{\infty} d z z^{-2 s} \frac{\partial}{\partial z} \log \left(1+\left(1+z^{2}\right)^{-\frac{1}{2}}\right), \\
& A_{-}(s) \equiv \sum_{n=3}^{\infty} n^{-(2 s-2)} \frac{(\sin \pi s)}{\pi} \int_{0}^{\infty} d z z^{-2 s} \frac{\partial}{\partial z} \log \left(\frac{1-\left(1+z^{2}\right)^{-\frac{1}{2}}}{z^{2}}\right)
\end{aligned}
$$

where $z^{2}$ in the denominator of the argument of the log arises, in Eq. (50), from the extra $z^{-2}$ in the prefactor $z^{-\beta_{-}(n)}$ in the definition (45). Moreover, the second pair of round brackets in Eq. (46) contributes $\sum_{j=1}^{\infty} A_{j, \pm}(s)$, having defined

$$
A_{j, \pm}(s) \equiv \sum_{n=3}^{\infty} n^{-(2 s+j-2)} \frac{(\sin \pi s)}{\pi} \int_{0}^{\infty} d z z^{-2 s} \frac{\partial}{\partial z} T_{j, \pm}(\tau(z)),
$$

where, from the formulae

$$
\begin{gathered}
T_{1, \pm}=p_{1, \pm} \\
T_{2, \pm}=p_{2, \pm}-\frac{1}{2} p_{1, \pm}^{2} \\
T_{3, \pm}=p_{3, \pm}-p_{1, \pm} p_{2, \pm}+\frac{1}{3} p_{1, \pm}^{3},
\end{gathered}
$$


we find

$$
\begin{gathered}
T_{1, \pm}=-\frac{3}{8} \tau \pm \frac{1}{2} \tau^{2}-\frac{5}{24} \tau^{3}, \\
T_{2, \pm}=-\frac{3}{16} \tau^{2} \pm \frac{3}{8} \tau^{3}+\frac{1}{8} \tau^{4} \mp \frac{5}{8} \tau^{5}+\frac{5}{16} \tau^{6}, \\
T_{3, \pm}=-\frac{21}{128} \tau^{3} \pm \frac{3}{8} \tau^{4}+\frac{509}{640} \tau^{5} \mp \frac{25}{12} \tau^{6}+\frac{21}{128} \tau^{7} \pm \frac{15}{8} \tau^{8}-\frac{1105}{1152} \tau^{9},
\end{gathered}
$$

and hence, in general,

$$
T_{j, \pm}(\tau)=\sum_{a=j}^{3 j} f_{a}^{(j, \pm)} \tau^{a} .
$$

We therefore find, from the first line of Eq. (44), contributions to the generalized $\zeta$-function, from terms in round brackets in Eq. (46), equal to

$$
\chi_{A}^{ \pm}(s)=\omega_{0}(s) F_{0}^{ \pm}(s)+\sum_{j=1}^{\infty} \omega_{j}(s) F_{j}^{ \pm}(s),
$$

where, for all $\lambda=0, j\left(\zeta_{R}\right.$ and $\zeta_{H}$ being the Riemann and Hurwitz $\zeta$ functions, respectively),

$\omega_{\lambda}(s) \equiv \sum_{n=3}^{\infty} n^{-(2 s+\lambda-2)}=\zeta_{H}(2 s+\lambda-2 ; 3)=\zeta_{R}(2 s+\lambda-2)-1-2^{-(2 s+\lambda-2)}$,

while, from Eqs. (49)-(51),

$$
\begin{gathered}
F_{0}^{+}(s) \equiv \frac{(\sin \pi s)}{\pi} \int_{0}^{\infty} d z z^{-2 s} \frac{\partial}{\partial z} \log \left(1+\left(1+z^{2}\right)^{-\frac{1}{2}}\right), \\
F_{0}^{-}(s) \equiv-2 \frac{(\sin \pi s)}{\pi} \int_{0}^{\infty} d z \frac{z^{-(2 s-1)}}{\left(1+z^{2}\right)}-F_{0}^{+}(s)=-1-F_{0}^{+}(s), \\
F_{j}^{ \pm}(s) \equiv \frac{(\sin \pi s)}{\pi} \sum_{a=j}^{3 j} L^{ \pm}(s, a, 0) f_{a}^{(j, \pm)},
\end{gathered}
$$

having set (this general definition will prove useful later, and arises from a more general case, where $\tau^{a}$ is divided by the $b$-th power of $(1 \pm \tau)$ in Eq. $(58))$

$L^{ \pm}(s, a, b) \equiv \int_{0}^{1} \tau^{2 s+a}(1-\tau)^{-s}(1+\tau)^{-s}\left( \pm b(1 \pm \tau)^{-b-1}-a \tau^{-1}(1 \pm \tau)^{-b}\right) d \tau$. 
Moreover, on considering

$$
L_{0}^{+}(s) \equiv \frac{\pi}{\sin \pi s} F_{0}^{+}(s),
$$

and changing variable from $z$ to $\tau$ therein, all $L$-type integrals above can be obtained from

$$
Q(\alpha, \beta, \gamma) \equiv \int_{0}^{1} \tau^{\alpha}(1-\tau)^{\beta}(1+\tau)^{\gamma} d \tau
$$

In particular, we will need

$$
\begin{gathered}
L_{0}^{+}(s)=-Q(2 s,-s,-s-1), \\
L^{+}(s, a, b)=b Q(2 s+a,-s,-s-b-1)-a Q(2 s+a-1,-s,-s-b),
\end{gathered}
$$

where, from the integral representation of the hypergeometric function, one has (Gradshteyn and Ryzhik [28])

$$
Q(\alpha, \beta, \gamma)=\frac{\Gamma(\alpha+1) \Gamma(\beta+1)}{\Gamma(\alpha+\beta+2)} F(-\gamma, \alpha+1 ; \alpha+\beta+2 ;-1) .
$$

For example, explicitly,

$$
L_{0}^{+}(s)=-\frac{\Gamma(2 s+1) \Gamma(1-s)}{\Gamma(s+2)} F(s+1,2 s+1 ; s+2 ;-1) .
$$

Now we exploit Eqs. (45), (46) and (59) to write

$$
\begin{aligned}
\zeta_{A}^{+}(s) & =\chi_{A}^{+}(s)+\frac{(\sin \pi s)}{\pi} \sum_{n=3}^{\infty} n^{-(2 s-2)} \int_{0}^{\infty} d z\left[\frac{z^{-(2 s-1)}}{2\left(1+z^{2}\right)}\right. \\
& \left.+n z^{-(2 s+1)}\left(\sqrt{1+z^{2}}-1\right)\right] .
\end{aligned}
$$

Hence we find

$$
\zeta_{A}^{+}(0)=\lim _{s \rightarrow 0}\left[\omega_{0}(s) F_{0}^{+}(s)+\sum_{j=1}^{\infty} \omega_{j}(s) F_{j}^{+}(s)+\left(\zeta_{A}^{+}(s)-\chi_{A}^{+}(s)\right)\right] .
$$

The first limit in Eq. (72) is immediately obtained by noting that

$$
\lim _{s \rightarrow 0} L_{0}^{+}(s)=-\log (2),
$$

and hence

$$
\lim _{s \rightarrow 0} \omega_{0}(s) F_{0}^{+}(s)=\lim _{s \rightarrow 0}\left[\zeta_{H}(2 s-2 ; 3) \frac{(\sin \pi s)}{\pi} L_{0}^{+}(s)\right]=0 .
$$


To evaluate the second limit in Eq. (72), we use

$$
\lim _{s \rightarrow 0} L^{+}(s, a, 0)=-1,
$$

and bear in mind that $\omega_{j}(s)$ is a meromorphic function with first-order pole, as $s \rightarrow 0$, only at $j=3$ by virtue of the limit

$$
\lim _{y \rightarrow 1}\left[\zeta_{R}(y)-\frac{1}{(y-1)}\right]=\gamma
$$

Hence we find (see coefficients in Eq. (57))

$$
\begin{aligned}
\lim _{s \rightarrow 0} \sum_{j=1}^{\infty} \omega_{j}(s) F_{j}^{+}(s) & =\lim _{s \rightarrow 0} \frac{(\sin \pi s)}{\pi} \sum_{j=1}^{\infty} \omega_{j}(s)\left[\sum_{a=j}^{3 j} L^{+}(s, a, 0) f_{a}^{(j,+)}\right] \\
& =-\frac{1}{2} \sum_{a=3}^{9} f_{a}^{(3,+)}=-\frac{1}{720}
\end{aligned}
$$

while, from Eqs. (71) and (69),

$$
\begin{aligned}
\lim _{s \rightarrow 0}\left(\zeta_{A}^{+}(s)-\chi_{A}^{+}(s)\right) & =\lim _{s \rightarrow 0}\left(\frac{1}{4} \zeta_{H}(2 s-2 ; 3)+\frac{1}{4 \sqrt{\pi}} \frac{\Gamma\left(s-\frac{1}{2}\right)}{\Gamma(s+1)} \zeta_{H}(2 s-3 ; 3)\right) \\
& =-\frac{5}{4}+\frac{1079}{240} .
\end{aligned}
$$

We therefore find, with the same algorithms as in Ref. 27,

$$
\begin{gathered}
\zeta_{A}^{+}(0)=-\frac{5}{4}+\frac{1079}{240}-\frac{1}{2} \sum_{a=3}^{9} f_{a}^{(3,+)}=\frac{146}{45}, \\
\zeta_{A}^{-}(0)=-\frac{5}{4}+\frac{1079}{240}+5-\frac{1}{2} \sum_{a=3}^{9} f_{a}^{(3,-)}=\frac{757}{90} .
\end{gathered}
$$

These results have been double-checked by using also the powerful analytic technique in Ref. 25.

\section{Further spectral asymptotics: elliptic and non-elliptic parts}

As a next step, the second line of Eq. (44) suggests considering $\zeta$-functions having the integral representation (using again the Cauchy theorem and 
rotation of contour as in Eq. (45))

$$
\begin{aligned}
& \zeta_{B}^{ \pm}(s) \equiv \frac{(\sin \pi s)}{\pi} \sum_{n=3}^{\infty} n^{-(2 s-2)} \\
& \int_{0}^{\infty} d z z^{-2 s} \frac{\partial}{\partial z} \log \left[z^{-\beta_{ \pm}(n)}\left(z n I_{n}^{\prime}(z n)+\left(\frac{z^{2} n^{2}}{2} \pm n\right) I_{n}(z n)\right)\right]
\end{aligned}
$$

To begin, we exploit again the uniform asymptotic expansion of modified Bessel functions and their first derivatives to find (cf. Eq. (46))

$$
z n I_{n}^{\prime}(z n)+\left(\frac{z^{2} n^{2}}{2} \pm n\right) I_{n}(z n) \sim \frac{n^{2}}{2 \sqrt{2 \pi n}} \frac{e^{n \eta}}{\sqrt{\tau}}\left(\frac{1}{\tau}-\tau\right)\left(1+\sum_{k=1}^{\infty} \frac{r_{k, \pm}(\tau)}{n^{k}}\right)
$$

where we have (bearing in mind that $u_{0}=v_{0}=1$ )

$$
r_{k, \pm}(\tau) \equiv u_{k}(\tau)+\frac{2 \tau}{\left(1-\tau^{2}\right)}\left(\left(v_{k-1}(\tau) \pm \tau u_{k-1}(\tau)\right),\right.
$$

for all $k \geq 1$. Hereafter we set

$$
\Omega \equiv \sum_{k=1}^{\infty} \frac{r_{k, \pm}(\tau(z))}{n^{k}}
$$

and rely upon the formula

$$
\log (1+\Omega) \sim \sum_{k=1}^{\infty}(-1)^{k+1} \frac{\Omega^{k}}{k}
$$

to evaluate the uniform asymptotic expansion (cf. Eq. (48))

$$
\log \left(1+\sum_{k=1}^{\infty} \frac{r_{k, \pm}(\tau(z))}{n^{k}}\right) \sim \sum_{k=1}^{\infty} \frac{R_{k, \pm}(\tau(z))}{n^{k}} .
$$

The formulae yielding $R_{k, \pm}$ from $r_{k, \pm}$ are exactly as in Eqs. (52)-(54), with $T$ replaced by $R$ and $p$ replaced by $r$ (see, however, comments below Eq. (90)). Hence we find, bearing in mind Eq. (83),

$$
\begin{gathered}
R_{1, \pm}=(1 \mp \tau)^{-1}\left(\frac{17}{8} \tau \mp \frac{1}{8} \tau^{2}-\frac{5}{24} \tau^{3} \pm \frac{5}{24} \tau^{4}\right) \\
R_{2, \pm}=(1 \mp \tau)^{-2}\left(-\frac{47}{16} \tau^{2} \pm \frac{15}{8} \tau^{3}-\frac{21}{16} \tau^{4} \pm \frac{3}{4} \tau^{5}-\frac{1}{16} \tau^{6} \mp \frac{5}{8} \tau^{7}+\frac{5}{16} \tau^{8}\right)
\end{gathered}
$$




$$
\begin{aligned}
R_{3, \pm} & =(1 \mp \tau)^{-3}\left(\frac{1721}{384} \tau^{3} \mp \frac{441}{128} \tau^{4}+\frac{597}{320} \tau^{5} \mp \frac{1033}{960} \tau^{6}+\frac{239}{80} \tau^{7}\right. \\
& \left.\mp \frac{28}{5} \tau^{8}+\frac{2431}{576} \tau^{9} \pm \frac{221}{192} \tau^{10}-\frac{1105}{384} \tau^{11} \pm \frac{1105}{1152} \tau^{12}\right)
\end{aligned}
$$

and therefore

$$
R_{j, \pm}(\tau(z))=(1 \mp \tau)^{-j} \sum_{a=j}^{4 j} C_{a}^{(j, \pm)} \tau^{a}
$$

where, unlike what happens for the $T_{j, \pm}$ polynomials, the exponent of $(1 \mp \tau)$ never vanishes. Note that, at $\tau=1$ (i.e. $z=0$ ), our $r_{k,+}(\tau)$ and $R_{k,+}(\tau)$ are singular. Such a behaviour is not seen for any of the strongly elliptic boundary-value problems [8]. This technical difficulty motivates our efforts below and is interpreted by us as a clear indication of the lack of strong ellipticity proved, on general ground, in Ref. 18.

The $\zeta_{B}^{-}(s)$ function is more easily dealt with. It indeed receives contributions from terms in round brackets in Eq. (82) equal to (cf. Eq. (50) and bear in mind that $\beta_{-}-\beta_{+}=2$ in Eq. (81))

$$
\begin{aligned}
B_{-}(s) & \equiv \sum_{n=3}^{\infty} n^{-(2 s-2)} \frac{(\sin \pi s)}{\pi} \int_{0}^{\infty} d z z^{-2 s} \frac{\partial}{\partial z} \log \left(\frac{\frac{1}{\tau(z)}-\tau(z)}{z^{2}}\right) \\
& =\omega_{0}(s) \frac{(\sin \pi s)}{\pi} \int_{0}^{\infty} d z z^{-2 s} \frac{\partial}{\partial z} \log \frac{1}{\sqrt{1+z^{2}}}=-\frac{1}{2} \omega_{0}(s),
\end{aligned}
$$

and $\sum_{j=1}^{\infty} B_{j,-}(s)$, having defined, with $\lambda=0, j$ (cf. Eq. (51))

$$
\begin{gathered}
\omega_{\lambda}(s) \equiv \sum_{n=3}^{\infty} n^{-(2 s+\lambda-2)}=\zeta_{H}(2 s+\lambda-2 ; 3), \\
B_{j,-}(s) \equiv \omega_{j}(s) \frac{(\sin \pi s)}{\pi} \int_{0}^{\infty} d z z^{-2 s} \frac{\partial}{\partial z} R_{j,-}(\tau(z)) .
\end{gathered}
$$

On using the same method as in Sec. 3, the formulae (81)-(93) lead to

$$
\zeta_{B}^{-}(0)=-\frac{5}{4}+\frac{1079}{240}+\frac{5}{2}-\frac{1}{16} \sum_{a=3}^{12} C_{a}^{(3,-)}=\frac{206}{45},
$$

a result which agrees with a derivation of $\zeta_{B}^{-}(0)$ relying upon the method of Ref. 25.

Although we have stressed after Eq. (90) the problems with the $\zeta_{B}^{+}(s)$ part, for the moment let us proceed formally in the same way as above. 
Thus we define, in analogy to Eq. (91),

$$
B_{+}(s) \equiv \omega_{0}(s) \frac{(\sin \pi s)}{\pi} \int_{0}^{\infty} d z z^{-2 s} \frac{\partial}{\partial z} \log \left(\frac{1}{\tau(z)}-\tau(z)\right),
$$

and, in analogy to Eq. (93),

$$
B_{j,+}(s) \equiv \omega_{j}(s) \frac{(\sin \pi s)}{\pi} \int_{0}^{\infty} d z z^{-2 s} \frac{\partial}{\partial z} R_{j,+}(\tau(z)) .
$$

In order to make the presentation as transparent as possible, we write out the derivatives of $R_{j,+}$. On changing integration variable from $z$ to $\tau$ we define

$$
C_{j}(\tau) \equiv \frac{\partial}{\partial \tau} R_{j,+}(\tau)
$$

and we find the following results:

$$
\begin{gathered}
C_{1}(\tau)=(1-\tau)^{-2}\left(\frac{17}{8}-\frac{1}{4} \tau-\frac{1}{2} \tau^{2}+\frac{5}{4} \tau^{3}-\frac{5}{8} \tau^{4}\right) \\
C_{2}(\tau)=(1-\tau)^{-3}\left(-\frac{47}{8} \tau+\frac{45}{8} \tau^{2}-\frac{57}{8} \tau^{3}+\frac{51}{8} \tau^{4}-\frac{21}{8} \tau^{5}-\frac{33}{8} \tau^{6}+\frac{45}{8} \tau^{7}\right. \\
\left.-\frac{15}{8} \tau^{8}\right) \\
C_{3}(\tau)=(1-\tau)^{-4}\left(\frac{1721}{128} \tau^{2}-\frac{441}{32} \tau^{3}+\frac{1635}{128} \tau^{4}-\frac{163}{16} \tau^{5}+\frac{1545}{64} \tau^{6}-\frac{227}{4} \tau^{7}\right. \\
\left.+\frac{4223}{64} \tau^{8}-\frac{221}{16} \tau^{9}-\frac{5083}{128} \tau^{10}+\frac{1105}{32} \tau^{11}-\frac{1105}{128} \tau^{12}\right)
\end{gathered}
$$

so that the general expression of $C_{j}(\tau)$ reads as

$$
C_{j}(\tau)=(1-\tau)^{-j-1} \sum_{a=j-1}^{4 j} K_{a}^{(j)} \tau^{a}, \forall j=1, \ldots, \infty .
$$

These formulae engender a $\zeta_{B}^{+}(0)$ which can be defined, after change of variable from $z$ to $\tau$, by splitting the integral with respect to $\tau$, in the integral representation of $\zeta_{B}^{+}(s)$, according to the identity

$$
\int_{0}^{1} d \tau=\int_{0}^{\mu} d \tau+\int_{\mu}^{1} d \tau
$$

and taking the limit as $\mu \rightarrow 1$ after having evaluated the integral. More precisely, since the integral on the left-hand side is independent of $\mu$, we can choose $\mu$ small on the right-hand side so that, in the interval $[0, \mu]$ (and 
only there!), we can use the uniform asymptotic expansion of the integrand where the negative powers of $(1-\tau)$ are harmless. Moreover, independence of $\mu$ also implies that, after having evaluated the integrals on the righthand side, we can take the $\mu \rightarrow 1$ limit. Within this framework, the limit as $\mu \rightarrow 1$ of the second integral on the right-hand side yields vanishing contribution to the asymptotic expansion of $\zeta_{B}^{+}(s)$.

With this caveat, on defining (cf. (66))

$$
Q_{\mu}(\alpha, \beta, \gamma) \equiv \int_{0}^{\mu} \tau^{\alpha}(1-\tau)^{\beta}(1+\tau)^{\gamma} d \tau,
$$

we obtain the representations

$$
\begin{aligned}
B_{+}(s) & =-\omega_{0}(s) \frac{(\sin \pi s)}{\pi}\left[-Q_{\mu}(2 s,-s-1,-s)+Q_{\mu}(2 s,-s,-s-1)\right. \\
& \left.-Q_{\mu}(2 s-1,-s,-s)\right] \\
B_{j,+}(s) & =-\omega_{j}(s) \frac{(\sin \pi s)}{\pi} \sum_{a=j-1}^{4 j} K_{a}^{(j)} Q_{\mu}(2 s+a,-s-j-1,-s) .
\end{aligned}
$$

The relevant properties of $Q_{\mu}(\alpha, \beta, \gamma)$ can be obtained by observing that this function is nothing but a hypergeometric function of two variables [28], i.e.

$$
Q_{\mu}(\alpha, \beta, \gamma)=\frac{\mu^{\alpha+1}}{\alpha+1} F_{1}(\alpha+1,-\beta,-\gamma, \alpha+2 ; \mu,-\mu)
$$

In detail, a summary of results needed to consider the limiting behaviour of $\zeta_{B}^{+}(s)$ as $s \rightarrow 0$ is

$$
\begin{gathered}
\omega_{0}(s) \frac{(\sin \pi s)}{\pi} \sim-5 s+\mathrm{O}\left(s^{2}\right), \\
\omega_{j}(s) \frac{(\sin \pi s)}{\pi} \sim \frac{1}{2} \delta_{j, 3}+\tilde{b}_{j, 1} s+\mathrm{O}\left(s^{2}\right), \\
\lim _{\mu \rightarrow 1} Q_{\mu}(2 s,-s-1,-s) \sim-\frac{1}{s}+\mathrm{O}\left(s^{0}\right), \\
\lim _{\mu \rightarrow 1} Q_{\mu}(2 s,-s,-s-1) \sim \log (2)+\mathrm{O}(s), \\
\lim _{\mu \rightarrow 1} Q_{\mu}(2 s-1,-s,-s) \sim \frac{1}{2 s}+\mathrm{O}(s),
\end{gathered}
$$


18

$$
\begin{aligned}
& \lim _{\mu \rightarrow 1} Q_{\mu}(2 s+a,-s-j-1,-s) \\
= & \frac{\Gamma(-j-s) \Gamma(a+2 s+1)}{\Gamma(a-j+s+1)}{ }_{2} F_{1}(a+2 s+1, s, a-j+s+1 ;-1) \\
\sim & \frac{b_{j,-1}(a)}{s}+b_{j, 0}(a)+\mathrm{O}(s)
\end{aligned}
$$

where

$$
\begin{gathered}
\tilde{b}_{j, 1}=-1-2^{2-j}+\zeta_{R}(j-2)\left(1-\delta_{j, 3}\right)+\gamma \delta_{j, 3}, \\
b_{j,-1}(a)=\frac{(-1)^{j+1}}{j !} \frac{\Gamma(a+1)}{\Gamma(a-j+1)}\left(1-\delta_{a, j-1}\right),
\end{gathered}
$$

and we only strictly need $b_{3,0}(a)$ which, unlike the elliptic cases studied earlier, now depends explicitly on $a$ and is given by ( $\psi$ being the standard notation for the logarithmic derivative of the $\Gamma$-function)

$$
\begin{aligned}
b_{3,0}(a) & =\frac{1}{6} \frac{\Gamma(a+1)}{\Gamma(a-2)}\left[-\log (2)-\frac{1}{4}\left(6 a^{2}-9 a+1\right) \frac{\Gamma(a-2)}{\Gamma(a+1)}+2 \psi(a+1)\right. \\
& -\psi(a-2)-\psi(4)] .
\end{aligned}
$$

Remarkably, the coefficient of $\frac{1}{s}$ in the small- $s$ behaviour of the generalized $\zeta$-function $\zeta_{B}^{+}(s)$ is zero because it is equal to

$$
\lim _{s \rightarrow 0} s \zeta_{B}^{+}(s)=\sum_{a=2}^{12} b_{3,-1}(a) K_{a}^{(3)}=\frac{1}{6} \sum_{a=3}^{12} a(a-1)(a-2) K_{a}^{(3)},
$$

which vanishes by virtue of the rather peculiar general property

$$
\sum_{a=j}^{4 j} \frac{\Gamma(a+1)}{\Gamma(a-j+1)} K_{a}^{(j)}=\sum_{a=j}^{4 j} \prod_{l=0}^{j-1}(a-l) K_{a}^{(j)}=0, \forall j=1, \ldots, \infty,
$$

and hence we find eventually

$$
\begin{aligned}
\zeta_{B}^{+}(0) & =-\frac{5}{4}+\frac{1079}{240}+\frac{5}{2}-\frac{1}{2} \sum_{a=2}^{12} b_{3,0}(a) K_{a}^{(3)}-\sum_{j=1}^{\infty} \tilde{b}_{j, 1} \sum_{a=j-1}^{4 j} b_{j,-1}(a) K_{a}^{(j)} \\
& =\frac{5}{4}+\frac{1079}{240}+\frac{599}{720}=\frac{296}{45}
\end{aligned}
$$

because the infinite sum on the first line of Eq. (117) vanishes by virtue of Eqs. (113) and (116), and exact cancellation of $\log (2)$ terms is found to occur by virtue of Eq. (116). 
To cross-check our analysis, we use Eq. (83) to evaluate

$$
r_{k,+}(\tau)-r_{k,-}(\tau)=\frac{4 \tau^{2}}{\left(1-\tau^{2}\right)} u_{k-1}(\tau)
$$

and hence we find

$$
\begin{gathered}
R_{1,+}=R_{1,-}+\frac{4 \tau^{2}}{\left(1-\tau^{2}\right)}, \\
R_{2,+}=R_{2,-}+\frac{4 \tau^{2}}{\left(1-\tau^{2}\right)}\left(u_{1}-\frac{2 \tau^{2}}{\left(1-\tau^{2}\right)}-R_{1,-}\right), \\
R_{3,+}=R_{3,-}+\frac{4 \tau^{2}}{\left(1-\tau^{2}\right)}\left(u_{2}-\frac{4 \tau^{2}}{\left(1-\tau^{2}\right)} u_{1}-u_{1} R_{1,-}-R_{2,-}+\frac{4 \tau^{2}}{\left(1-\tau^{2}\right)} R_{1,-}\right) \\
+\frac{64}{3} \frac{\tau^{6}}{\left(1-\tau^{2}\right)^{3}}+\frac{2 \tau^{2}}{\left(1-\tau^{2}\right)} R_{1,-}^{2},
\end{gathered}
$$

and so on. This makes it possible to evaluate $B_{j,+}(s)-B_{j,-}(s)$ for all $j=1,2, \ldots \infty$. Only $j=3$ contributes to $\zeta_{B}^{ \pm}(0)$ (see below) and we find

$$
\begin{aligned}
& B_{3,+}(s)-B_{3,-}(s)=-\omega_{3}(s) \frac{(\sin \pi s)}{\pi} \\
& \cdot \lim _{\mu \rightarrow 1} \int_{0}^{\mu} d \tau \tau^{2 s}(1-\tau)^{-s}(1+\tau)^{-s} \frac{\partial}{\partial \tau}\left(R_{3,+}-R_{3,-}\right) .
\end{aligned}
$$

The derivative in the integrand on the right-hand side of Eq. (122) reads as

$$
\frac{\partial}{\partial \tau}\left(R_{3,+}-R_{3,-}\right)=(1-\tau)^{-4}(1+\tau)^{-4}\left(80 \tau^{3}-24 \tau^{5}+32 \tau^{7}-8 \tau^{9}\right),
$$

and hence we can use again the definition (102) and the formula (105) to express (122) through the functions $Q_{\mu}(2 s+a,-s-4,-s-4)$, with $a=3,5,7,9$. This leads to

$$
\begin{aligned}
\zeta_{B}^{+}(0) & =\zeta_{B}^{-}(0)+B_{3,+}(0)-B_{3,-}(0) \\
& =\zeta_{B}^{-}(0)-\frac{1}{24} \sum_{l=1}^{4} \frac{\Gamma(l+1)}{\Gamma(l-2)}\left[\psi(l+2)-\frac{1}{(l+1)}\right] \kappa_{2 l+1}^{(3)} \\
& =\frac{206}{45}+2=\frac{296}{45}
\end{aligned}
$$

where $\kappa_{2 l+1}^{(3)}$ are the four coefficients on the right-hand side of (123). Regularity of $\zeta_{B}^{+}(s)$ at the origin is guaranteed because $\lim _{s \rightarrow 0} s \zeta_{B}^{+}(s)$ is proportional to

$$
\sum_{l=1}^{4} \frac{\Gamma(l+1)}{\Gamma(l-2)} \kappa_{2 l+1}^{(3)}=0,
$$


which is a particular case of the peculiar spectral cancellation (cf. (116))

$$
\sum_{a=a_{\min }(j)}^{a_{\max }(j)} \frac{\Gamma\left(\frac{(a+1)}{2}\right)}{\Gamma\left(\frac{(a+1)}{2}-j\right)} \kappa_{a}^{(j)}=0,
$$

where $a$ takes both odd and even values. The case $j=3$ is simpler because then only $\kappa_{a}^{(j)}$ coefficients with odd $a$ are non-vanishing.

Remaining contributions to $\zeta(0)$, being obtained from strongly elliptic sectors of the boundary-value problem, are easily found to agree with the results in Ref. 19, i.e.

$$
\begin{gathered}
\zeta(0)[\text { transverse traceless modes }]=-\frac{278}{45}, \\
\zeta(0)[\text { coupled vector modes }]=\frac{494}{45}, \\
\zeta(0)[\text { decoupled vector mode }]=-\frac{15}{2}, \\
\zeta(0)\left[\text { scalar modes }\left(a_{1}, e_{1} ; a_{2}, b_{2}, e_{2}\right)\right]=-17, \\
\zeta(0)[\text { scalar ghost modes }]=-\frac{149}{45}, \\
\zeta(0)[\text { vector ghost modes }]=\frac{77}{90}, \\
\zeta(0)[\text { decoupled ghost mode }]=\frac{5}{2} .
\end{gathered}
$$

Our full $\zeta(0)$ is therefore, from (79), (80), (94), (117), (126)-(132), $\zeta(0)=$ $\frac{142}{45}$.

\section{Concluding remarks}

We have studied the analytically continued eigenvalue conditions for metric perturbations on the Euclidean four-ball, in the presence of boundary conditions completely invariant under infinitesimal diffeomorphisms in the de Donder gauge and with the $\alpha$ parameter set to 1 in Eq. (10). This has made it possible to prove that only one sector of the scalar-mode determinant is responsible for lack of strong ellipticity of the boundary-value problem (see second line of Eq. (44) and the analysis in Secs. 3 and 4). The first novelty with respect to the work in Ref. 18 is a clear separation 
of the elliptic and non-elliptic sectors of spectral asymptotics for Euclidean quantum gravity. We have also shown that one can indeed obtain a regular $\zeta$-function asymptotics at small $s$ in the non-elliptic case by virtue of the remarkable identity (116). Our prescription for the $\zeta(0)$ value differs from the result first obtained in Ref. 19, where, however, neither the strong ellipticity issue [18] nor the non-standard spectral asymptotics of our Sec. 4 had been considered.

As far as we can see, the issues raised by our results are as follows.

(i) The integral representation (81) is legitimate because the second line of Eq. (44) corresponds to the eigenvalue conditions, for $n \geq 3$,

$$
F_{B}^{ \pm}(n, x) \equiv J_{n}^{\prime}(x)+\left(-\frac{x}{2} \pm \frac{n}{x}\right) J_{n}(x)=0 .
$$

For both choices of sign in front of $\frac{n}{x}$, if $x_{i}$ is a root, then so is $-x_{i}$, with positive eigenvalue $E_{i}=x_{i}^{2}$ (having set the 3 -sphere radius $q=1$ for simplicity). For any fixed $n$, there is a countable infinity of roots $x_{i}$ and they grow approximately linearly with the integer $i$ counting such roots. The function $F_{B}^{ \pm}$admits therefore a canonical-product representation (Ahlfors [29]) which ensures that the integral representation (81) reproduces the standard definition of generalized $\zeta$-function, i.e.

$$
\zeta(s) \equiv \sum_{E_{k}>0} d\left(E_{k}\right) E_{k}^{-s},
$$

where $d\left(E_{k}\right)$ is the degeneracy of the eigenvalue $E_{k}$.

(ii) Even though the lack of strong ellipticity implies that the functional trace of the heat semigroup no longer exists, and hence the Mellin transform relating $\zeta$-function to integrated heat kernel cannot be exploited, it remains possible to define the functional determinant of the operator $P$ acting on metric perturbations. For this purpose, a weaker assumption provides a sufficient condition, i.e. the existence of a sector in the complex plane free of eigenvalues of the leading symbol of $P$ (Seeley [30]). Note also that, if one looks at the $A_{1}$ heat-kernel coefficient for boundary conditions involving tangential derivatives [8], it is exactly for the ball that the potentially divergent pieces involving the extrinsic curvature in $A_{1}$ cancel. Thus, on the Euclidean ball cancellations take place that maybe could explain why $\zeta(0)$ is finite. This might be therefore a very particular result for the ball.

(iii) By virtue of standard recurrence relations among Bessel functions, the eigenvalue conditions (133) are equivalent to studying the eigenvalue 
conditions

$$
\widetilde{F}_{B}^{ \pm}(n, x)=J_{n}(x) \mp \frac{2}{x} J_{n-1}(x)=0,
$$

where the eigenvalues $E(i, n, \pm)$ are obtained by squaring up the roots $x(i, n, \pm)$. The equation for $\widetilde{F}_{B}^{-}(n, x)$ can be further re-expressed in the form

$$
\left(1+\frac{4 n}{x^{2}}\right) J_{n}(x)-\frac{2}{x} J_{n-1}(x)=0
$$

The functions $\widetilde{F}_{B}^{ \pm}$differ therefore by one term only, and this term gets small as $x$ gets larger. The numerical analysis confirms indeed that a $\rho(i, n)$ positive and much smaller than 1 exists such that one can write (Esposito et al. [31])

$$
E(i, n,+)=E(1, n,+) \delta_{i, 1}+E(i-1, n,-)(1+\rho(i, n))\left(1-\delta_{i, 1}\right),
$$

for all $n \geq 3$ and for all $i \geq 1$.

(iv) The remarkable factorization of eigenvalue conditions, with resulting isolation of elliptic part of spectral asymptotics (transverse-traceless, vector and ghost modes, all modes in finite-dimensional sub-spaces and three of the four equations for scalar modes), suggests trying to re-assess functional integrals on manifolds with boundary, with the hope of being able to obtain unique results from the non-elliptic contribution. If this cannot be achieved, the two alternatives below should be considered again.

(v) Luckock boundary conditions (Luckock [32]), which engender BRSTinvariant amplitudes but are not diffeomorphism invariant [15]. They have already been applied by Moss and Poletti [12, 33].

(vi) Non-local boundary conditions that lead to surface states in quantum cosmology and pseudo-differential operators on metric and ghost modes (Marachevsky and Vassilevich, Esposito [34]). Surface states are particularly interesting since they describe a transition from quantum to classical regime in cosmology entirely ruled by the strong ellipticity requirement, while pseudo-differential operators are a source of technical complications.

There is therefore encouraging evidence in favour of Euclidean quantum gravity being able to drive further developments in quantum field theory, quantum cosmology and spectral asymptotics (see early mathematical papers by Grubb [35], Gilkey and Smith [36]) in the years to come. 


\section{Appendix: Olver expansions}

In Secs. 3 and 4 we use the uniform asymptotic expansion of modified Bessel functions $I_{\nu}$ first found by Olver [37]:

$$
I_{\nu}(z \nu) \sim \frac{e^{\nu \eta}}{\sqrt{2 \pi \nu}\left(1+z^{2}\right)^{\frac{1}{4}}}\left(1+\sum_{k=1}^{\infty} \frac{u_{k}(\tau)}{\nu^{k}}\right)
$$

where

$$
\tau \equiv\left(1+z^{2}\right)^{-\frac{1}{2}}, \eta \equiv\left(1+z^{2}\right)^{\frac{1}{2}}+\log \left(\frac{z}{1+\sqrt{1+z^{2}}}\right) .
$$

This holds for $\nu \rightarrow \infty$ at fixed $z$. The polynomials $u_{k}(\tau)$ can be found from the recurrence relation [27]

$$
u_{k+1}(\tau)=\frac{1}{2} \tau^{2}\left(1-\tau^{2}\right) u_{k}^{\prime}(\tau)+\frac{1}{8} \int_{0}^{\tau} d \rho\left(1-5 \rho^{2}\right) u_{k}(\rho),
$$

starting with $u_{0}(\tau)=1$. Moreover, the first derivative of $I_{\nu}$ has the following uniform asymptotic expansion at large $\nu$ and fixed $z$ :

$$
I_{\nu}^{\prime}(z \nu) \sim \frac{e^{\nu \eta}}{\sqrt{2 \pi \nu}} \frac{\left(1+z^{2}\right)^{\frac{1}{4}}}{z}\left(1+\sum_{k=1}^{\infty} \frac{v_{k}(\tau)}{\nu^{k}}\right),
$$

with the $v_{k}$ polynomials determined from the $u_{k}$ according to [27]

$$
v_{k}(\tau)=u_{k}(\tau)+\tau\left(\tau^{2}-1\right)\left[\frac{1}{2} u_{k-1}(\tau)+\tau u_{k-1}^{\prime}(\tau)\right],
$$

starting with $v_{0}(\tau)=u_{0}(\tau)=1$.

\section{Acknowledgments}

We are grateful to Bernhelm Booß-Bavnbek and Krysztof Wojciechowski for their kind invitation and encouragement. Moreover, we are indebted to Ivan Avramidi for inspiration provided by previous collaboration with some of us and by continuous correspondence, and to Gerd Grubb for enlightening correspondence. K. Kirsten is grateful to the Baylor University Research Committee, to the Max-Planck-Institute for Mathematics in the Sciences (Leipzig, Germany) and to the INFN for financial support. The work of G. Esposito and K. Kirsten has been partially supported also by PRIN SINTESI. The work of A.Yu. Kamenshchik was partially supported by the Russian Foundation for Basic Research under the Grant No. 02-02-16817 and by the Scientific School Grant No. 2338.2003.2 


\section{References}

1. D. Deutsch and P. Candelas, Boundary effects in quantum field theory, Phys. Rev. D 20 (1979), 3063-3080.

2. B. S. DeWitt, "The Global Approach to Quantum Field Theory", Oxford University Press, Oxford, 2003.

3. J. B. Hartle and S. W. Hawking, Wave function of the universe, Phys. Rev. D 28 (1983), 2960-2975; S. W. Hawking, The quantum state of the universe, Nucl. Phys. B 239 (1984), 257-276.

4. S. W. Hawking, The boundary conditions for gauged supergravity, Phys. Lett. B 126 (1983), 175-177.

5. A. Abouelsaood, C. G. Callan, C. R. Nappi and S. A. Yost, Open strings in background gauge fields, Nucl. Phys. B 280 (1987), 599624 .

6. M. Bordag, U. Mohideen and V. M. Mostepanenko, New developments in the Casimir effect, Phys. Rep. 353 (2001), 1-205 (quantph/0106045); K. Milton, "The Casimir Effect: Physical Manifestations of Zero-Point Energy", World Scientific, River Edge, 2001; V. V. Nesterenko, G. Lambiase and G. Scarpetta, Calculation of the Casimir energy at zero and finite temperature: some recent results, Riv. Nuovo Cim. 27, Ser. 4, Num. 6 (2004), 1-74 (hep-th/0503100).

7. P. Gilkey, The spectral geometry of a Riemannian manifold, J. Diff. Geom. 10 (1975), 601-618.

8. K. Kirsten, "Spectral Functions in Mathematics and Physics", CRC Press, Boca Raton, 2001.

9. D. V. Vassilevich, Heat kernel expansion: user's manual, Phys. Rep. 388 (2003), 279-360 (hep-th/0306138).

10. I. G. Moss, Boundary terms in the heat kernel expansion, Class. Quantum Grav. 6 (1989), 759-765.

11. I. G. Avramidi, "Heat Kernel and Quantum Gravity", Lecture Notes in Physics m64, Springer-Verlag, Berlin, 2000.

12. G. Esposito, A. Yu. Kamenshchik, I. V. Mishakov and G. Pollifrone, Euclidean Maxwell theory in the presence of boundaries. II, Class. Quantum Grav. 11 (1994), 2939-2950 (gr-qc/9506061); I. G. Moss and S. Poletti, Conformal anomalies on Einstein spaces with boundary, Phys. Lett. B 333 (1994), 326-330 (gr-qc/9405044).

13. G. Tsoupros, Conformal anomalies for interacting scalar fields on curved manifolds with boundary, Int. J. Mod. Phys. A 20 (2005), 10271064 (hep-th/0311100); Conformal anomaly for free scalar propagation on curved bounded manifolds, Gen. Rel. Grav. 37 (2005), 399-406 (hep-th/0409163).

14. G. Esposito, "Quantum Gravity, Quantum Cosmology and Lorentzian Geometries", Lecture Notes in Physics Vol. m12, Springer-Verlag, Berlin, 1994.

15. G. Esposito, A. Yu. Kamenshchik and G. Pollifrone, "Euclidean Quantum Gravity on Manifolds with Boundary", Fundamental Theories of 
Physics Vol. 85, Kluwer, Dordrecht, 1997.

16. A. O. Barvinsky, The wave function and the effective action in quantum cosmology: covariant loop expansion, Phys. Lett. B 195 (1987), 344-348.

17. I. G. Moss and P. Silva, BRST invariant boundary conditions for gauge theories, Phys. Rev. D 55 (1997), 1072-1078 (gr-qc/9610023).

18. I. G. Avramidi and G. Esposito, Gauge theories on manifolds with boundary, Commun. Math. Phys. 200 (1999), 495-543 (hepth/9710048); Lack of strong ellipticity in Euclidean quantum gravity, Class. Quantum Grav. 15 (1998), 1141-1152 (hep-th/9708163).

19. G. Esposito, A. Yu. Kamenshchik, I. V. Mishakov and G. Pollifrone, One-loop amplitudes in Euclidean quantum gravity, Phys. Rev. D 52 (1995), 3457-3465 (gr-qc/9504016).

20. I. G. Avramidi, G. Esposito and A. Yu. Kamenshchik, Boundary operators in Euclidean quantum gravity, Class. Quantum Grav. 13 (1996), 2361-2373 (hep-th/9603021).

21. K. Schleich, Semiclassical wave function of the universe at small three geometries, Phys. Rev. D 32 (1985), 1889-1898.

22. J. S. Dowker and K. Kirsten, Heat kernel coefficients for oblique boundary conditions, Class. Quantum Grav. 14 (1997), L169-L175 (hepth/9706129).

23. E. M. Lifshitz and I. M. Khalatnikov, Investigations in relativistic cosmology, Adv. Phys. 12 (1963), 185-249.

24. G. Esposito, A. Yu. Kamenshchik, I. V. Mishakov and G. Pollifrone, Gravitons in one-loop quantum cosmology. Correspondence between covariant and non-covariant formalisms, Phys. Rev. D 50 (1994), 63296337 (gr-qc/9506066).

25. A. O. Barvinsky, A. Yu. Kamenshchik and I. P. Karmazin, One-loop quantum cosmology: $\zeta$-function technique for the Hartle-Hawking wave function of the universe, Ann. Phys. (N.Y.) 219 (1992), 201242.

26. G. Esposito, G. Fucci, A. Yu. Kamenshchik and K. Kirsten, Spectral asymptotics of Euclidean quantum gravity with diff-invariant boundary conditions, Class. Quantum Grav. 22 (2005), 957-974 (hepth/0412269).

27. M. Bordag, E. Elizalde and K. Kirsten, Heat kernel coefficients of the Laplace operator on the D-dimensional ball, J. Math. Phys. 37 (1996), 895-916 (hep-th/9503023); M. Bordag, J. S. Dowker and K. Kirsten, Heat kernels and functional determinants on the generalized cone, Commun. Math. Phys. 182 (1996), 371-394 (hep-th/9602089).

28. I. S. Gradshteyn and I. M. Ryzhik, "Table of Integrals, Series and Products", Academic Press, New York, 1965.

29. L. V. Ahlfors, "Complex Analysis", McGraw-Hill, New York, 1966.

30. R. T. Seeley, Complex powers of an elliptic operator, Am. Math. Soc. Proc. Symp. Pure Math. 10 (1967), 288-307.

31. G. Esposito, G. Fucci, A. Yu. Kamenshchik and K. Kirsten, A non- 
singular one-loop wave function of the universe from a new eigenvalue asymptotics in quantum gravity, JHEP 0509 (2005), 063, 1-16 (hepth/0507264).

32. H. C. Luckock, Mixed boundary conditions in quantum field theory, $J$. Math. Phys. 32 (1991), 1755-1766.

33. I. G. Moss and S. Poletti, Boundary conditions for quantum cosmology, Nucl. Phys. B 341 (1990), 155-161.

34. V. N. Marachevsky and D. V. Vassilevich, A diffeomorphism-invariant eigenvalue problem for metric perturbations in a bounded region, Class. Quantum Grav. 13 (1996), 645-652 (gr-qc/9509051); G. Esposito, Non-local boundary conditions in Euclidean quantum gravity, Class. Quantum Grav. 16 (1999), 1113-1126 (gr-qc/9806057); New kernels in quantum gravity, Class. Quantum Grav. 16 (1999), 39994010 (hep-th/9906169); Boundary operators in quantum field theory, Int. J. Mod. Phys. A 15 (2000), 4539-4555 (hep-th/0001086).

35. G. Grubb, Properties of normal boundary problems for elliptic evenorder systems, Ann. Sc. N. Sup. Pisa, Ser. IV, 1 (1974), 1-61.

36. P. B. Gilkey and L. Smith, The twisted index problem for manifolds with boundary, J. Diff. Geom. 18 (1983), 393-444.

37. F. W. J. Olver, On Bessel functions of large order, Phil. Trans. R. Soc. Lond. A 247 (1954), 328-368. 